\title{
Research on Composite Design of Modern Urban Square
}

\author{
Jun $\mathrm{Yi}^{1, *}$ \\ ${ }^{1}$ Wenhua College, Wuhan, Hubei, China \\ *Corresponding author. Email: 276807055@qq.com
}

\begin{abstract}
In view of the "image projects" and "vanity projects" in the design of modern urban square, as well as the problems of single function, large scale and low utilization rate, this paper sums up the development trend of modern urban square, puts forward the compound concept, and expounds the connotation of compound from the aspects of land compound, traffic compound, function compound, space compound, environmental system compound and historical and cultural compound. In order to improve the design of some "face-saving projects" of urban square, the composite design of modern urban square is formed, which can provide reference for the innovation and sustainable development of modern urban square design.
\end{abstract}

Keywords: Composite Design, Modern urban square, Function compound.

\section{INTRODUCTION}

Aristotle once said: "in order to live, people gather in cities; in order to live better, they stay in cities."[1] This sentence incisively reveals the essential characteristics of urban space, that is, agglomeration and concentration. As a concentrated urban space system and an important part of urban external space, urban square can not only connect buildings and roads, but also allow people to relax and stay here. It not only inherits the tradition and history of the city, meets the spiritual function needs of the city, but also is the human space presenting urban characteristics and style. Its gathering and dispersing effect can naturally bring lasting vitality to the city, which depends on the diversity, continuity, richness and composition of the urban square.

\section{THE CONNOTATION OF "COMPOUND"}

What is compound? In short, it is combination. ${ }^{1}$ In the "composite" design of modern urban square, it is to comprehensively organize and improve the activity space of citizens' communication. Through the integration of composite means and a series of design language expressions, it can efficiently use the urban public space, carry the diverse activities and multiple functional needs of people at all levels. Through the interpenetration, infiltration and integration of the land use, function, ecology and culture of urban space, the urban public space can be diversified in function, pleasant in space and diversified in ecology, meeting people's physiological and psychological needs. [2]

\section{THE COMPOSITE DESIGN CONTENT OF MODERN URBAN SQUARE}

\subsection{Land Compound}

The ultimate goal of compound urban square is consistent with the development goal of urban construction. According to the sustainable urban design principles, it should not waste limited natural resources. And the first is the compound use of land resources, that is, the intensification of land resources. [3] ("Figure 1")

1. $\mathrm{Bu} \mathrm{Yu}$. "Research on the Compound Function of University Campus", Postgraduate Thesis Network, 2006. 


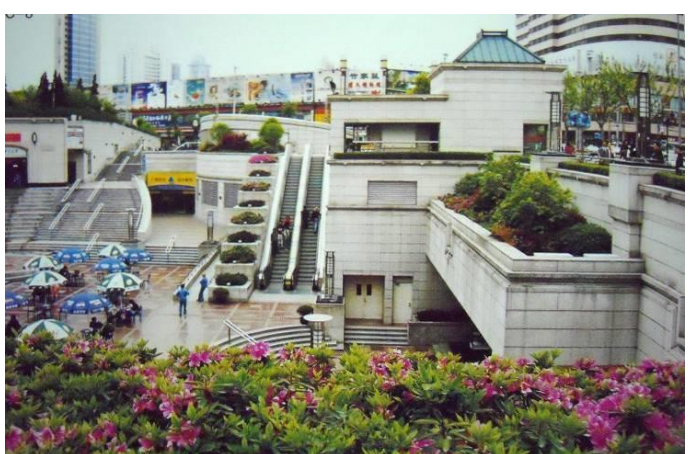

Figure 1 Sinking Square, Jing'an Temple, Shanghai.

\subsection{Function Compound}

With the rapid development of the city and urban life, it is required that the functions of the urban square should be rich and diverse. It should not only meet the leisure needs of local residents, but also meet the aesthetic needs of foreign tourists. At the same time, the functions should be coordinated to form an efficient, compact and orderly development mode of urban space. [4]

\subsubsection{The Functional Zoning Compound}

The functional zoning compound is not for all the urban squares, but for the square with broad vision and large scale. In order to break the monotony of the grand and open square, the functional zoning of the square should be compounded, so as to achieve rich functional changes.

\subsubsection{The Internal Compound of Each Functional Zoning}

The internal compound of functional divisions mainly refers to the combination of different kinds of functions, such as the combination of commercial function and gathering function, the combination of gathering function and leisure function, and the combination of leisure function and commercial function. In other words, each function does not have to exist in a single way, but can choose to compound with each other, achieving the maximum utilization of resources. ("Figure 2", "Figure 3")

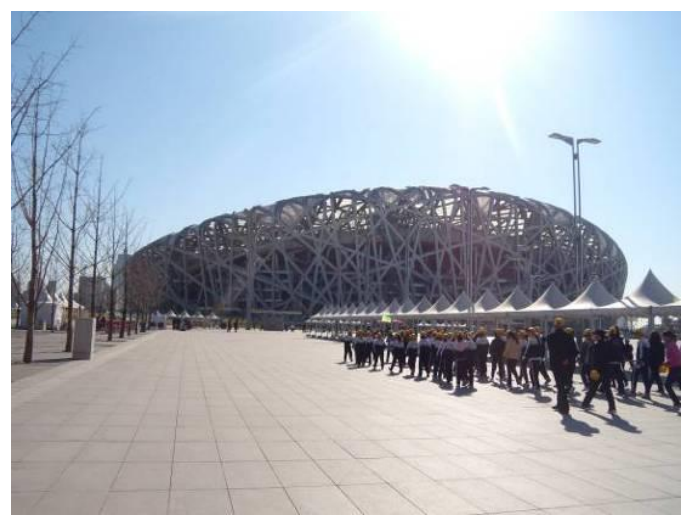

Figure 2 The architectural landscape of Beijing Olympic Square.

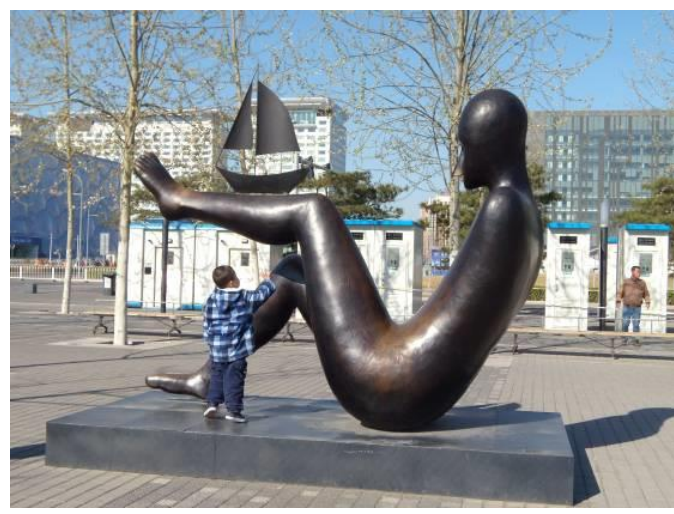

Figure 3 The sculpture landscape of Beijing Olympic Square.

\subsection{Space Compound}

The modern urban square has the problems of large scale and single space type. The only way to solve this problem is to compound the space. [5]

\subsubsection{The Compound of Urban Square Space Types}

\subsubsection{The Compound of Space Types}

The compound of square space types is the inevitable embodiment of the humanization of square. Through the composite design, the square provides the open space of mass gathering and the leisure space.

\subsubsection{The Compound of Different Space Types}

The compound of space types refers to the combination of different space types, such as the combination of open space and semi-open space, the combination of semi-open space and private space. In this way, it can meet various needs of 
different people in different regions and attract more users.

\subsubsection{The Compound of Spatial Scale of Urban Square}

As one of the standards to measure aesthetics, scale refers to people's evaluation and standard of the size of objective things. The object of scale is the size of objective things, while the subject is human. ("Figure 4", "Figure 5")

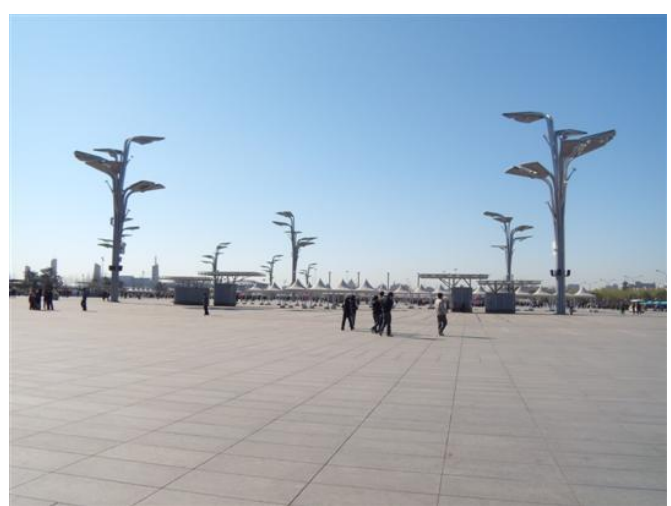

Figure 4 The open space of Beijing Olympic Square.

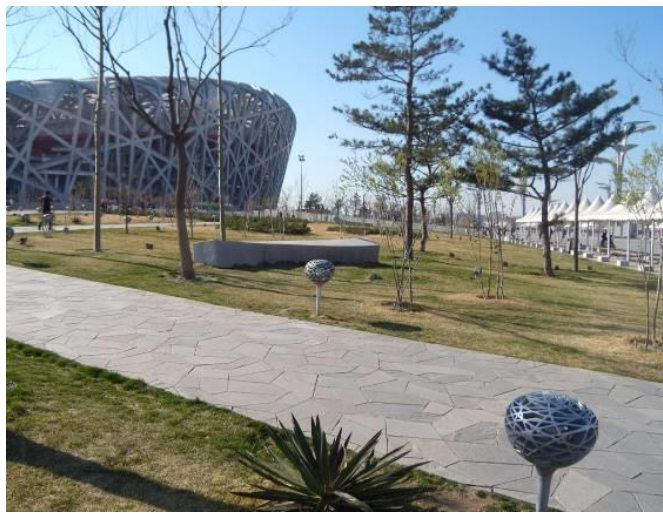

Figure 5 The private space of Beijing Olympic Square.

\subsection{The Compound in Natural Ecological Environment System}

The natural ecological environment system is an important part of the urban form system, which brings vitality to the city with its soft characteristics and full of vitality. Also, it is the most basic source of shaping the urban form. In order to build an environment-friendly city, it is required to do a good job in the design of the ecological environment system when constructing every road, every region and boundary of the city, as well as every important node and sign object. The problems of waste of land resources, low greening rate and groundwater seepage caused by the largescale hard pavement in traditional urban square should be solved one by one in the design of urban square. [6] ("Figure 6")

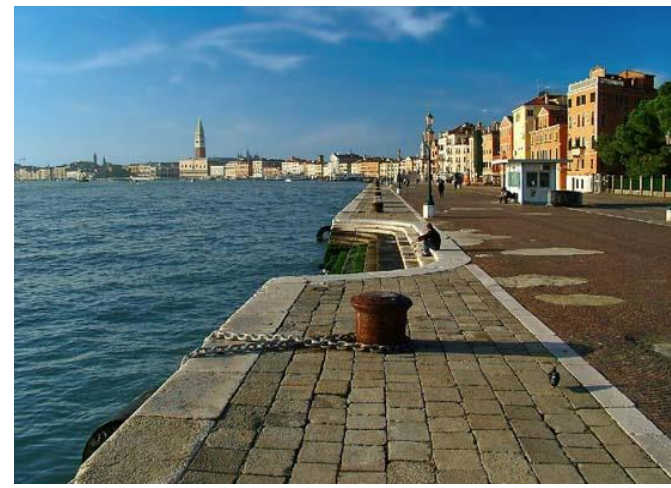

Figure 6 Water view of the Piazza San Marco.

\subsection{The Compound of History and Culture}

The culture of a city is produced by people and their related habits and activities. In the long-term life and production process, people have accumulated a lot of rich languages and ethnic characteristics. Due to some historical reasons, various ethnic groups and regions have different historical cultures, such as the carnival in Venice, the bullfighter in Spain, and the paper-cutting art in China. [7] ("Figure 7")

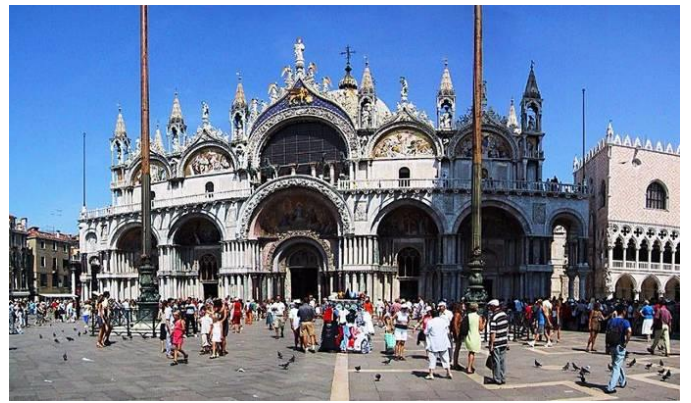

Figure 7 The masked ball at the Piazza San Marco.

\subsection{The Conditions for the "Compound" of Modern Urban Square}

The compound of modern urban square is inevitable, but we can't generalize it all in the planning and design. Some urban squares need to be compounded, while others don't, which depends on the nature, scale, development characteristics and development prospect of the urban square itself. 


\subsubsection{Super Large-scale Square}

The scale of a urban square is formulated according to the nature of the city and the demand proportion of the population, not for the "images" and "faces". Many urban square designers and city leaders do not design squares according to this principle, and then the scale of modern urban square is out of line with the demand of the urban population. Finally, there is few people in the square, or the square can't meet people's living needs.

\subsubsection{The Function of Squares Cannot Meet People's Needs}

With the continuous changes of people's needs, the functions of modern urban square present a diversified development trend. The urban squares should meet people's needs of large-scale gathering and leisure activities in daily life. That is to say, some urban squares with single function can no longer meet people's needs. They need the composite design of functions to transform the single square into a compound square.

There are four types of "compound" in modern urban squares.

- Juxtaposition type

The internal compound of leisure functions, concentrate functions, producing compound effects and improving space efficiency.

- Principal and subordinate type

gathering function and leisure function

- Complementary type

The functions complement each other to form a whole. Leisure function and commercial function

- Series type

It is to compound functional units with the conversion relationship of interoperability and continuity to promote the convenience and efficiency of the system.

\section{ANALYSIS ON COMPOSITE DESIGN OF BINSHUI SQUARE IN SHAOHAI XINCHENG, JIAOZHOU, QINGDAO}

The public open green belt of Shaohai Xincheng, Jiaozhou, Qingdao is located on the west bank of South Lake in Shaohai Xincheng, which is the first impression from the urban area to Shaohai
Xincheng via Hong Kong Road and shows the image and achievements of the new city.

\subsection{Opportunities and Challenges for "Composite" Design of Shaohai Xincheng, Jiaozhou, Qingdao}

\subsubsection{Opportunities for "Composite" Design of Shaohai Xincheng}

Binshui Square has good service and traffic conditions. Also, it is close to cities and airports and can provide basic traffic conditions for composite design.

Shaohai Xincheng has large tracts of undeveloped land, which is conducive to the development of multiple activities and functions, and has great potential for diversification.

Shaohai Xincheng has a large area of water, which is conducive to the development of a variety of entertainment activities and improves the quality design of the development. At the same time, the coastline and lake space are conducive to the enrichment of spatial levels.

Shaohai Xincheng has rich local history and cultural heritage, which is conducive to the exchange and development of various cultures.

\subsubsection{Challenges for "Composite" Design of Shaohai Xincheng}

Some rigid lakeshores that have been built have certain restrictions on design.

The degree of soil salinization is large, which affects the selection and survival rate of plants.

The shape of designed land is long and narrow, with the same width, which is slightly monotonous.

\section{2 "Composite" Design Orientation of Shaohai Xincheng}

\subsubsection{In View of the Challenges, the "Composite" Design Countermeasures of Shaohai Xincheng Are Proposed}

According to the landscape zoning, different lakeshore treatment methods are adopted to enrich the levels and increase the interest. For example, natural stone and grass slope revetment, grass slope revetment and wetland planting revetment are adopted in the leisure area and plant viewing area 
of the square, and hard revetment is adopted in the Binshui Square.

With the use of the method of adjusting measures to local conditions, it tries to choose the local tree species resistant to salt and alkali, and combine the soil and water quality to make design.

Due to the limitation of the topography of Shaohai Xincheng, multi-scale and diverse space and uses are created through plant landscaping.

\subsubsection{The Design Principle of Binshui Square of Shaohai Xincheng - Compound}

Taking the lake as the city card, it is to set up a wide green public space and take the urban public space with high quality and high starting point as the highlight, so as to attract the attention of tourists. With the use of the characteristics of the lake space, it creates multi-level and interesting landscape nodes and provides rich visual experience.

It is necessary to make full use of the advantages of land resources and transportation resources to create diversified land use patterns and perfect modern transportation system.

It emphasizes the harmony of vision, body and mind with the society, strengthens the functions of leisure and entertainment, and meets the leisure needs of people in the business district and community.

Based on the local historical and cultural traditions, various entertainment and tourism activities should be carried out, and the regional historical and cultural connotation should be reflected, so as to make it a modern city full of vitality.

Following the design principle of ecosystem, it is required to focus on nature and ecology, attract birds and insects, and form an ecosystem.

\subsubsection{The "Composite" Design Method of Shaohai Xincheng}

The main scenic spots of Binshui square of South Lake landscape project of Shaohai Xincheng in Jiaozhou, Qingdao are: entrance square, parking lot, footpath around the lake, wharf, office and exhibition of Shaohai Xincheng, shrub pattern, Binshui landscape square, gathering and performance square, garden path, characteristic structure or landscape wall, art gallery, memorial sculpture, lamp post, color trees, etc. ("Figure 8")

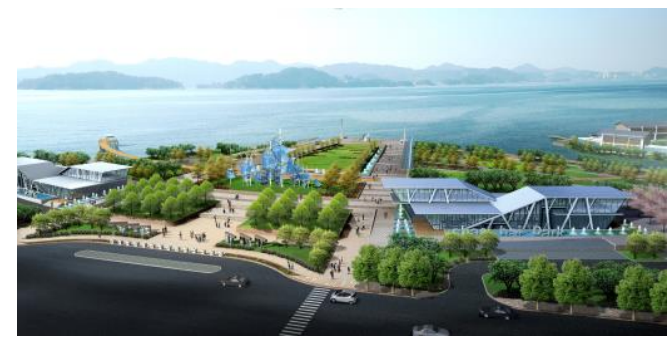

Figure 8 The bird's-eye view of Binshui square.

In terms of traffic and land compound, there is a good traffic environment to facilitate the citizens to reach. By compound means, the monotonous phenomenon of narrow and long Shaohai area with the same width should be avoided, and measures should be taken according to local conditions as far as possible. The visual effect of Jiaozhou Bay Highway should be emphasized to show the image of the new city.

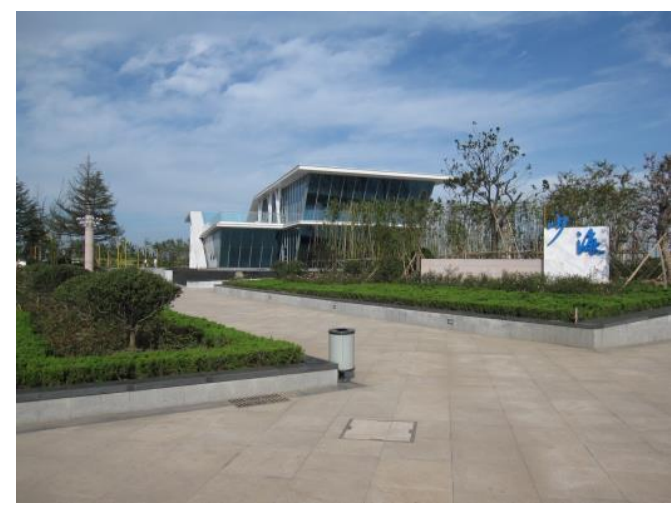

Figure 9 The landscape of Shaohai Xincheng Square at the entrance.

In "Figure 9", it strengthens the function of leisure and entertainment in terms of function and space compound to meet the leisure needs of people in the business district and community, creates multi-scale and diverse activity space and uses through plant landscaping, adopts different lakeside treatment methods according to the landscape zoning to enrich the landscape layers, increase the interest of the landscape, attract the enthusiasm of urban residents to participate, and meet the multilevel use needs and psychological needs of contemporary people. 


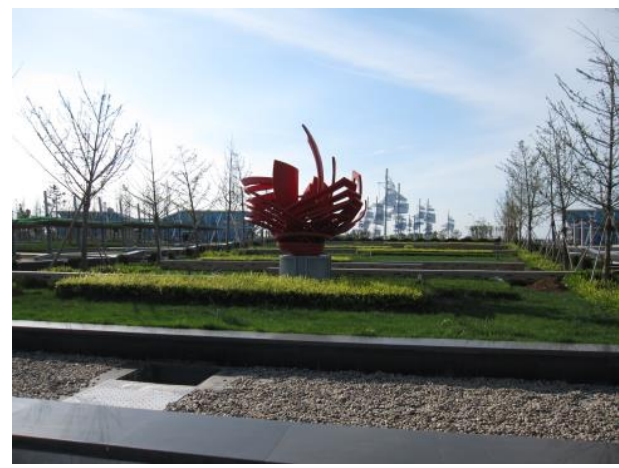

Figure 10 The sculpture landscape of Shaohai Xincheng Square.

In "Figure 10", nature and ecology are the main factors in the composite design of environmental ecosystem, and birds and insects are attracted to form an ecosystem, protecting the scenery of the sea. The revetment treatment of natural ecology makes the building elements on the lake and square achieve a state of symbiosis and prosperity. In "Figure 11", it reflects the connotation of history and culture in the compound of history and culture, and uses the artistic language forms and plant species with local characteristics to show the city features with distinctive style. ("Figure 12")

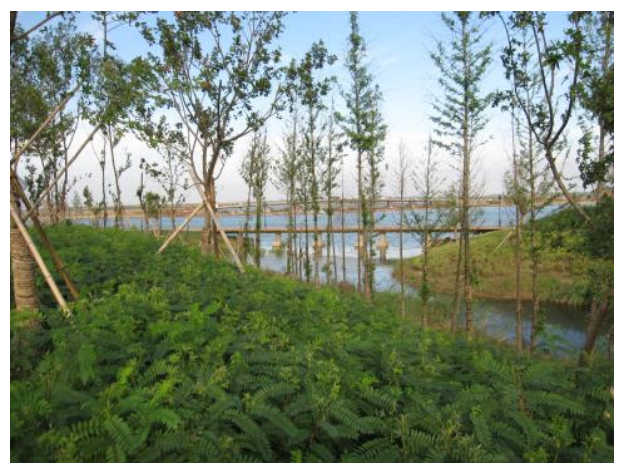

Figure 11 Ecological landscape of Shaohai Xincheng Square.

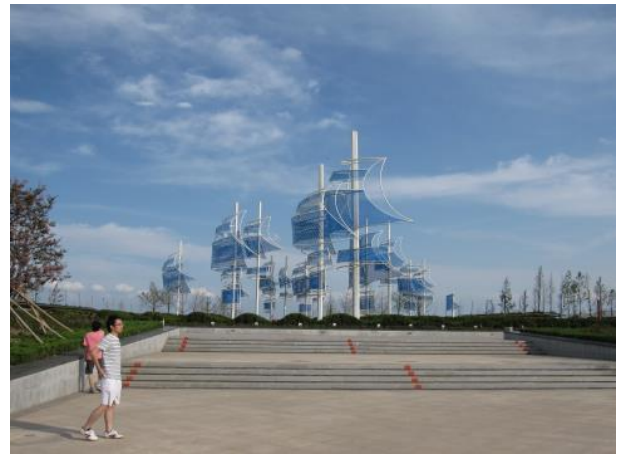

Figure 12 The sailing landscape of Shaohai Xincheng Square.

\section{CONCLUSION}

This paper analyzes the existing problems and development trend of modern urban square. Based on people's physiological, psychological and behavioral needs, the concept of "compound" of urban square is proposed.

The problems to be further studied in this paper are as follows.

First, it is necessary to study how to realize the composite design of modern urban square, and how to carry out the overall planning and control of the square from the perspective of the city, so as to realize the harmonious coexistence of people and the square.

Second, it should study how to retain people in modern urban square, which mainly depends on the rich activity function and space of square. Therefore, the author proposes the "composite" concept, hoping to create a three-dimensional square, that is, the combination of spaces above the ground, on ground and below ground. When considering the spaces, people should also consider the impact of modern rapid transportation on urban squares, such as overpass, rail transit, the distribution center of the lower subway and so on. These are what the compound design of modern square lacks.

Third, while improving the composite design theory, it is necessary to have a deep understanding of the living habits and psychological needs of local residents, and constantly explore and find ways to develop modern urban squares suitable for local people.

\section{AUTHORS' CONTRIBUTIONS} $\mathrm{Yi}$

This paper is independently completed by Jun

\section{REFERENCES}

[1] Jane Jacobs. "Life and Death in Big American Cities", Yilin Publishing House, 2005. (in Chinese)

[2] Yu Tao, Yang Yongsheng. "Modern Urban Waterscape Design and Construction", China City Press, 2003.1. (in Chinese)

[3] Jin Jun. "Ideal Landscape", Southeast University Press, 2003.1. (in Chinese) 
[4] Wang Peng. "Research on the Systematization of Urban Public Space", Southeast University Press, 2002. (in Chinese)

[5] Yu Baichun. "Extraordinary Urban Design Thoughts, Systems, Details", China Architecture Publishing House, 2008.11. (in Chinese)

[6] Hu Zhengfan. "Environmental Psychology and Environment - Behavioral Research", China Architecture Publishing House, 2016. (in Chinese)

[7] Liu Jie. "The Integration of Urban Forms", Southeast University Press, 2004.10. (in Chinese) 